\title{
Morphing modes of mobility in natural and engineered systems
}

\author{
R. J. Lock, R. Vaidyanathan \& S. Burgess \\ Department of Mechanical Engineering, \\ University of Bristol, Bristol, BS8 1TR, UK
}

\begin{abstract}
Autonomous vehicle utility has reached a plateau due to mobility constraints on the current generation of units in the field. Of particular note is the inability of existing robotic systems to manoeuvre in more than one substrate (e.g. land, air, water). Although no mature engineering examples exist today, many animals possess this capacity. Utility for robots often reflects a similar design space as small animals; multiple locomotion modes would represent a generational leap in their capability. Flight could allow a vehicle to approach a general target area, while crawling or swimming locomotion would enable otherwise unachievable tasks (e.g. close inspection, surveillance, sampling, etc.). The goal of our research is to develop a scalable architecture, drawing on inspiration from nature, for autonomous systems with the capacity for morphing modes of mobility. While much research has been performed into biological mechanisms of locomotion in a single medium, the tradeoffs, potential synergies, and basic measures of performance supporting natural mobility in several substrates has yet to be rigorously investigated from a design perspective. In this work we report modelling of the functional, physical, and operational architectures for a candidate set of animals with multiple modes of locomotion, with specific focus on the scalability of avian designs. Modelling of key parameters is used to demonstrate their effectiveness under specific engineering measures of performance. In the longer term, this work is envisaged to provide a foundation upon which to base the design of robotic systems capable of multiple modes of mobility as well as to analyze morphing locomotion modes in nature

Keywords: animal inspired locomotion mechanisms, morphing structures, flying and walking mobility, amphibious mobility, biologically inspired system architecting.
\end{abstract}




\section{Introduction}

In the natural world, animals commonly utilize multiple forms of (i.e. multimodal) locomotion, enabling the ability to function, in some capacity, within different environmental substrates. Currently, autonomous vehicles with the capacity to operate in more than one substrate have not reached mainstream design or use [1], and the highly novel projects that exist tend to lack scalability in the context of broader design. The premise of this research is that by analyzing morphologies of multi-modal locomotion in animals and understanding synergies between specific combinations, fundamental paradigms can be elucidated to provide a foundation for engineering design.

While overlapping mediums exist, natural modes of locomotion can broadly be decomposed into three categories; land, air and water. For each mode of locomotion, the predominant force that must be overcome to achieve required movement varies considerably (e.g. gravity and structural stress for land, drag and gravity for air, and drag (higher density) for water). It is clear the power requirements for different modes differ greatly, and the subsequent types of locomotion utilized in each will vary depending on the governing forces behind each medium. Understanding the way animals have optimized performance across multiple modes can offer insights of significant importance for future engineering design.

\section{Locomotion performance}

When considering multi-modal animal locomotion it is important to remember that the motivation behind natural behaviour is needs driven to supporting operations such as hunting, evasion, feeding and travel. Speed, acceleration, and endurance present conflicting measures of performance with a range of tradeoffs. For example, many predators have a slower top speed than their prey, but with a greater acceleration and timing, they may successfully hunt [2]. Prey have conversely developed greater manoeuvrability to evade attacks made by predators with quicker acceleration [2]. Fundamentally, all these measures relate to energy consumption. Consider, for example that penguins (Pygoscelis) consume energy approximately 60\% faster than turkeys (Meleagris) on land, despite having similar mass and locomotion speed [3]. Penguins (Eudyptula), however, only use 0.72 times the energy of ducks (Anas) of the same mass while swimming on the surface of water [4]. As with mobile robots, the predominant use of energy within animals is for locomotion; isolating synergies between morphologies is critical to enabling this capacity to artificial vehicles.

Considering the range of substrates to be traversed it is clear that no animal will excel at all of these. The animal optimizes tradeoffs between the different attributes of the locomotion modes based on its own measures of performance. By understanding how particular multi-modal animals have made this compromise, future engineering projects can adopt similar criteria. Table 1 
Table 1: Locomotion performance for various animals and engineering systems.

\begin{tabular}{|c|c|c|c|c|c|c|c|c|c|}
\hline & & Space & $\begin{array}{c}\text { High } \\
\text { Altitude }\end{array}$ & Air & $\begin{array}{l}\text { Water } \\
\text { Surface }\end{array}$ & $\begin{array}{l}\text { Under } \\
\text { water }\end{array}$ & Land & $\begin{array}{l}\text { Under } \\
\text { ground }\end{array}$ & $\begin{array}{l}\text { Total } \\
\text { score }\end{array}$ \\
\hline \multicolumn{10}{|l|}{ Nature } \\
\hline \multirow[t]{4}{*}{ Birds (Typical) } & Guillemot & & & $\mathrm{E}$ & $\mathrm{C}$ & $\mathrm{E}$ & $\mathrm{N}$ & & 9 \\
\hline & Black-legged kittiwake & & & $\mathrm{E}$ & $\mathrm{C}$ & $\mathrm{C}$ & $\mathrm{N}$ & & 8 \\
\hline & Puffin & & & $\mathrm{C}$ & $\mathrm{C}$ & $\mathrm{C}$ & $\mathrm{N}$ & $\mathrm{N}$ & 8 \\
\hline & Ruppell's Griffon vulture & & $\mathrm{C}$ & $\mathrm{C}$ & & & $\mathrm{N}$ & & 5 \\
\hline Insects & Water beetle & & & $\mathrm{C}$ & $\mathrm{E}$ & $\mathrm{N}$ & $\mathrm{C}$ & $\mathrm{N}$ & 9 \\
\hline \multirow{2}{*}{ Reptiles } & Crocodile & & & & $\mathrm{C}$ & $\mathrm{C}$ & $\mathrm{C}$ & & 6 \\
\hline & Paradise tree snake & & & & & & $\mathrm{E}$ & $\mathrm{N}$ & 4 \\
\hline Amphibians & Frog & & & & $\mathrm{C}$ & $\mathrm{C}$ & $\mathrm{C}$ & & 6 \\
\hline \multirow[t]{2}{*}{ Mammals } & Beaver & & & & $\mathrm{C}$ & $\mathrm{C}$ & $\mathrm{C}$ & $\mathrm{N}$ & 7 \\
\hline & Man & & & & $\mathrm{C}$ & $\mathrm{N}$ & $\mathrm{E}$ & & 6 \\
\hline Fish & Ray & & & $\mathrm{N}$ & & $\mathrm{E}$ & & $\mathrm{N}$ & 5 \\
\hline
\end{tabular}

\begin{tabular}{|l|l|l|l|l|l|l|l|l|l|}
\hline Engineering & Space shuttle & E & C & C & & & N & & \\
\hline \multirow{5}{*}{} & Sounding rocket & E & N & N & & & & & 5 \\
\cline { 2 - 11 } & Amphibious vehicles & & & & C & & C & & 4 \\
\cline { 2 - 10 } & Planetary lander & N & N & N & & & C & & 5 \\
\hline
\end{tabular}

summarizes locomotion performance for different animal types and environments, providing actual examples of animals within these groups.

For each category the animal has been given a rating of either expert (3), competent (2) or novice (1), labelled E, C and $\mathrm{N}$ respectively. Expert can be assumed to mean the animal performs well at several of the locomotion attributes, such as being fast and manoeuvrable and capable of performing many of the animal's main day to day tasks under these conditions. Competent refers to an environment that the animal has a moderate level of ability in and is able to perform relatively complex tasks. Finally, novice means the animal performs at a very basic level in these surroundings. Although the animal is utilizing this additional environment, they are by no means fully competent within it, and will typically only utilize the area to perform one basic task. It should be stressed that in this report we are principally interested in the animal's locomotive ability, focussing on techniques that could be utilised within engineering projects. In addition to the analysis of the animals, some engineering examples have been given that utilize multi-modal locomotion, and the same rating system has been applied to them. Birds and insects perform well in several modes of locomotion and offer the most potential for further analysis. Although birds do not always operate in a multi-modal fashion, they have, as a species, proven to be extremely competent in every substrate (barring space flight). Furthermore, avian designs have shown scalability in size that few other natural systems match. The common guillemot performs particularly well in both air and water, and was selected as a candidate creature for analysis of multiple locomotion modes.

\section{Locomotion and effectiveness in various substrates}

Now that the performance of multi-modal animals has been considered the actual types of locomotion used in the different mediums needs to be examined. Again the types of locomotion can be broken down into main categories; these can be seen in table 2 . The locomotion type is then cross-referenced with animal types 
that use that particular method. What is important to note is that multi-modal animals can utilize one of two options; firstly, morphology of one type of locomotion can be used in order to operate in different environments, with a level of adjustment made to accommodate the different conditions, or secondly use of two completely different techniques used in different environments.

From the table we can see that birds, insects and reptiles all utilize different types of locomotion with varying level of competence. We can also see that for land and on and underwater, there is a dominant type of locomotion.

Table 2: $\quad$ Types of locomotion.

\begin{tabular}{|c|c|c|c|c|c|c|c|c|c|}
\hline & & Birds & $\begin{array}{c}\text { Insec } \\
t\end{array}$ & Rept. & Amph & Fish & $\begin{array}{c}\text { Mamma } \\
1\end{array}$ & Man & Total \\
\hline \multirow{6}{*}{ Land } & Walking & $\mathrm{Y}$ & $\mathrm{Y}$ & $\mathrm{Y}$ & $\mathrm{Y}$ & & $\mathrm{Y}$ & $\mathrm{Y}$ & 6 \\
\hline & Running & $\mathrm{Y}$ & $\mathrm{Y}$ & $\mathrm{Y}$ & & & $\mathrm{Y}$ & $\mathrm{Y}$ & 5 \\
\hline & Tail Trst & & & $\mathrm{Y}$ & & & & & $\underline{1}$ \\
\hline & Snaking & & & $\mathrm{Y}$ & & & & & 1 \\
\hline & Jet prop. & & $\mathrm{Y}$ & & & & & & 1 \\
\hline & Hopping & $\mathrm{Y}$ & $\mathrm{Y}$ & & $\mathrm{Y}$ & & $\mathrm{Y}$ & $\mathrm{Y}$ & $\underline{5}$ \\
\hline \multirow[t]{2}{*}{ Air } & Flapping & $\mathrm{Y}$ & $\mathrm{Y}$ & & & & $\mathrm{Y}$ & & 3 \\
\hline & Gliding & $\mathrm{Y}$ & & $\mathrm{Y}$ & & & $\mathrm{Y}$ & & $\underline{3}$ \\
\hline & & & & & & & & & \\
\hline \multirow{4}{*}{$\begin{array}{l}\text { Under } \\
\text { water }\end{array}$} & Flapping & $\mathrm{Y}$ & $\mathrm{Y}$ & & & $\mathrm{Y}$ & $\mathrm{Y}$ & & $\underline{4}$ \\
\hline & Tail Trst & & & & & $\mathrm{Y}$ & & & $\overline{1}$ \\
\hline & Snaking & & & $\mathrm{Y}$ & & $\mathrm{Y}$ & & & $\underline{2}$ \\
\hline & Jet prop. & & & & & $\mathrm{Y}$ & & & $\underline{1}$ \\
\hline \multirow{4}{*}{$\begin{array}{l}\text { Water } \\
\text { surf }\end{array}$} & Paddling & $\mathrm{Y}$ & $\mathrm{Y}$ & $\bar{Y}$ & $\mathrm{Y}$ & & $\bar{Y}$ & $\mathrm{Y}$ & 6 \\
\hline & Flapping & $\mathrm{Y}$ & & & & & & & $\underline{1}$ \\
\hline & Snaking & & & $\mathrm{Y}$ & & & & & 1 \\
\hline & Skating & & $\mathrm{Y}$ & & & & & & 1 \\
\hline \multirow[t]{2}{*}{$\begin{array}{l}\text { Under } \\
\text { grnd }\end{array}$} & Burrow & Y & Y & Y & & & Y & & 4 \\
\hline & Total & $\underline{9}$ & $\underline{9}$ & $\underline{9}$ & $\underline{\underline{3}}$ & $\underline{4}$ & $\underline{8}$ & $\underline{4}$ & \\
\hline
\end{tabular}

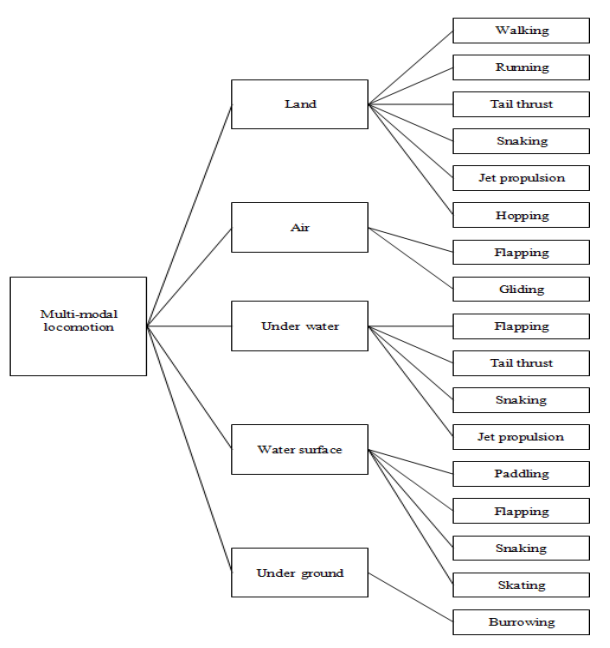

Figure 1: Generic model to help demonstrate effectiveness of specific multimodal animals. 
To help in determining which combinations of locomotion types work well together, it is important to establish levels of effectiveness and performance for each animal. Using the information from Table 2, a diagrammatic representation can be given to evaluate specific multi-modal locomotion. This model is shown in Figure 1.

\section{Environmental considerations}

In order to understand various locomotion modalities we must consider the operating environment in more detail. Table 3 summarises key features. It is clear each environment offers its own benefits and drawbacks; animals must optimize their performance so as to limit the negative implications and extending their operating range.

Table 3: $\quad$ Features of environment (ranges taken from typical animals).

\begin{tabular}{|c|c|c|c|c|c|}
\hline & Air & Water surface & Underwater & Land & Underground \\
\hline $\begin{array}{c}\text { Need for altitude } \\
\text { support }\end{array}$ & $\mathrm{Y}$ & $\mathrm{N}$ & $\mathrm{N}$ & $\mathrm{Y}$ & $\mathrm{N}$ \\
\hline $\begin{array}{c}\text { Resistance to } \\
\text { motion }\end{array}$ & Low & Medium & Medium & Low & High \\
\hline Coasting possible & $\mathrm{Y}$ & $\mathrm{Y}$ & $\mathrm{Y}$ & $\mathrm{N}$ & $\mathrm{N}$ \\
\hline $\begin{array}{c}\text { Body passively } \\
\text { supported (by } \\
\text { pressure) }\end{array}$ & $\mathrm{N}$ & $\mathrm{N}$ & $\mathrm{Y}$ & $\mathrm{N}$ & $\mathrm{Y}$ \\
\hline $\begin{array}{c}\text { Skill needed to } \\
\text { move }\end{array}$ & $\mathrm{Y}$ & $\mathrm{Y}$ & $\mathrm{N}$ & $\mathrm{Y}$ & $\mathrm{Y}$ \\
\hline $\begin{array}{c}\text { Range (one day, } \\
\text { miles) }\end{array}$ & $1000 \mathrm{~m}$ & Low & $500 \mathrm{~m}$ & $100 \mathrm{~m}$ & $0.01 \mathrm{~m}$ \\
\hline $\begin{array}{c}\text { Places of refuge } \\
\text { (protection) }\end{array}$ & $\mathrm{N}$ & $\mathrm{N}$ & $\mathrm{Y}$ & $\mathrm{Y}$ & $\mathrm{Y}$ \\
\hline $\begin{array}{c}\text { Food readily } \\
\text { available[0] }\end{array}$ & $\mathrm{Y}$ & $\mathrm{Y}$ & $\mathrm{Y}$ & $\mathrm{Y}$ & $\mathrm{Y}$ \\
\hline $\begin{array}{c}\text { Ability to stop/rest } \\
\text { (a) }\end{array}$ & $\mathrm{N}$ & $\mathrm{Y}$ & $\mathrm{N}$ & $\mathrm{Y}$ & $\mathrm{Y}$ \\
\hline
\end{tabular}

\section{Avian design focus}

Although it has been established that birds, insects and reptiles all operate in various environmental conditions, we have decided to focus the study on birds. By no means are we implying that insects and reptiles do not offer similar insights, but simply that birds appear to possess an intriguing set of beneficial characteristics to engineering design.

Firstly, in terms of scalability, birds offer a greater range of breeds capable of flight, operating between $1.5 \mathrm{~g}$ and $15 \mathrm{~kg}$. Comparing this with the insect's range of $1 \mathrm{ug}$ to $20 \mathrm{~g}$, the difference is clear. Although not always multi-modal, for every substrate, particular birds operate with an excellent level of competence. Ruppell's griffon vulture is capable of flying at altitudes of 37,000 feet [5], whilst the ostrich, weighing as much as $150 \mathrm{~kg}$ can achieve land speeds of $43 \mathrm{mph}$ at a sprint, and sustained speeds over long distances of $31 \mathrm{mph}$ [6]. Birds also 
demonstrate admirable competence through multiple mediums. As detailed in Table 1, the common guillemot is not only a very capable flyer, but actually morphs its wing shape so as to optimize performance underwater.

Therefore by utilizing multiple modes of locomotion, birds can make the most of the benefits specific to each environment. For example a bird could use flight to travel much greater distances than possible if using land locomotion. This could enable the bird to reach superior feeding areas, which would be otherwise unobtainable. The bird, such as the guillemot, could then completely change its mode of locomotion and dive under water in order to catch the desired food. This ability to utilize both modes of locomotion has a huge potential in engineering projects in areas from defence and military applications, to search and rescue operations.

\section{Multi-modal locomotion of the common guillemot}

The common guillemot (Uria aalge), also referred to as the common murre, is part of the Auk family, marine birds which can be found in the cooler parts of the northern seas [7]. They exhibit very interesting multi-modal locomotion abilities, but this does not come without compromise. In this section we shall examine the different types of locomotion used, looking into the interactions of the chosen technique with the various environment conditions as described in Table 3. The calculations performed are first order approximations at our current level of abstraction; more detail will be addressed in future research.

\subsection{Guillemots in air: Flapping}

Guillemots utilise their ability to fly in order to reach food sources that are far out at sea away from the coastal nesting area. Typically they fly $60 \mathrm{~km}$ out to sea during the pre-nesting season, and this value reduces to $20 \mathrm{~km}$ when chick rearing. However guillemots have been sited carrying fish in their mouths from as far as $70 \mathrm{~km}$ from the nesting site [7]. Their ability to fly allows the birds to seek out the prime areas for collecting fish, where they can then change the mode of locomotion in order to catch the food source.

Guillemots and other members of the Auk family have relatively large, stocky bodies in relation to their wing size, resulting in very high wing loading in flight. Pennycuick [8] reports this value is as high as $171 \mathrm{~N} / \mathrm{m}^{2}$ which is greater than that experienced by the wandering albatross despite having a weight over 8 times greater. Pennycuick [8] also explained this through analysis of the wings of the alcid family, of which the guillemot is a part. Alcid wings have reduced span and area compared to 'standard' seabird wings, but with little difference in the aspect ratio or the size and mass of the body. This reduction results in an increase in both gliding and flapping speeds for the common guillemot. The common guillemot had a mean observed airspeed of $19.1 \mathrm{~m} / \mathrm{s}$, the fastest out of all examined in [8]. In order to maintain this airspeed, guillemots must operate with a continuous high wing-beat frequency $(\sim 8.7 \mathrm{~Hz})[9]$. Consequently, they cannot flap-glide (as most sea birds do) and have limited ability at slow speeds. 
In flight, the guillemot must overcome external resistance in order to maintain steady horizontal motion. This can be broken down into three forms of drag that the bird is subjected to. These are induced drag due to the lift on the wings, profile drag due to the changes in wing pressure and skin friction, and parasitic drag due to the actual body of the bird.

Induced drag, profile drag and parasitic drag are modelled in (1)-(3), where $m$ is the mass of the bird (kg), $g$ is the gravitational constant, $b$ is the wing span (m), $U$ is the air speed $(\mathrm{m} / \mathrm{s}), \rho$ is the density of air $\left(\mathrm{kg} / \mathrm{m}^{3}\right), S$ is the wing area $\left(\mathrm{m}^{2}\right), S_{b}$ is the frontal area of the body $\left(\mathrm{m}^{2}\right)$ and $C_{D-P r o}$ and $C_{D-B o d}$ are the drag coefficients for the profile and body of the guillemot:

$$
D_{\text {Ind }}=2 \mathrm{~km}^{2} \mathrm{~g}^{2} /\left(\pi b^{2} U^{2} \rho\right)(1), D_{\mathrm{Pr} o}=0.5 \rho S U^{2} C_{D-\mathrm{P} \mathrm{r} o}(2), D_{\text {Par }}=0.5 \rho S_{b} U^{2} C_{D-B o d}
$$

Constants in these relations were taken from $[8,10,11]$.

Taking power as the product of drag force and average airspeed gives the total average power required for the guillemot to sustain horizontal flight:

$$
\begin{gathered}
P_{\text {Ind }}=2 \mathrm{~km}^{2} \mathrm{~g}^{2} /\left(\pi b^{2} U \rho\right)=5.74 \mathrm{~W}(4), P_{\mathrm{Pro}}=0.5 \rho S U^{3} C_{D-\operatorname{Pro}}=3.2 \mathrm{~W} \\
P_{\text {Par }}=0.5 \rho S_{b} U^{3} C_{D-P a r}=8.01 \mathrm{~W}(6), P_{\text {Sum }}=P_{\text {Ind }}+P_{\mathrm{Pr} o}+P_{\text {Par }}=16.96 \mathrm{~W}
\end{gathered}
$$

Due to the smaller wing size of the bird in comparison to its body size, the guillemot must flap at faster speeds than standard sea birds, and also travels at faster airspeeds. It can be seen that profile drag and parasitic drag are proportional to the square of the airspeed at which the bird is travelling, and as guillemots travel at a relatively high mean airspeed of $19.1 \mathrm{~m} / \mathrm{s}$ [8] these values of drag will be higher than those experienced in birds of similar body size but with larger wing areas.

\subsection{Guillemots under water: Flapping}

Guillemots change their mode of locomotion from air to water in order to hunt. They have been recorded to dive as deep as $100 \mathrm{~m}$, but they typically feed in depths between 20 to $50 \mathrm{~m}$ [7]. It is within this change of environment from air to water that the guillemots morph their locomotion modalities. They still use a flapping technique, but as water is approximately 800 times denser than air at sea level, the governing forces involved with the locomotion type changes considerably. When flapping underwater, guillemots change their wing shape so that they are strongly flexed, thus the effective surface area of the wing consists mainly of the primary feathers, with the remaining held close into the body, as shown in Figure 2.

Figure 2 demonstrates a clear change in wing-shape. In the denser water, far less area is required to produce the same amount of force to propel the bird, even when the bird is flapping slower [2]. While the wing beat frequency of the bird in the air was approximately $8.7 \mathrm{~Hz}$, when underwater, it is $1.9-2.8 \mathrm{~Hz}$ [11].

Additionally, whereas in the air where the inner portion of the wing is mainly used to help with supporting the weight of the bird, this is no longer needed as the animals buoyancy makes the body weightless [13]. This results in the guillemot being able to reduce its wing size to no more than the area that is used for producing the thrust. In actual fact when birds swim underwater they are 
subjected to forces lifting them up to the surface due to the air in their lungs and trapped within feathers. Some species of penguins produce greater thrust on the upstroke rather than the down stroke when swimming horizontally to overcome this buoyancy [14]. While guillemots appear to create thrust on the upstroke, they still rely on greater thrust on the down stroke when flying in the air. Figure 3 details wing positions during horizontal swimming by a guillemot, [11]. Figure 4 shows the angles of attack of the wings of the Humboldt penguin whilst swimming horizontally [2].

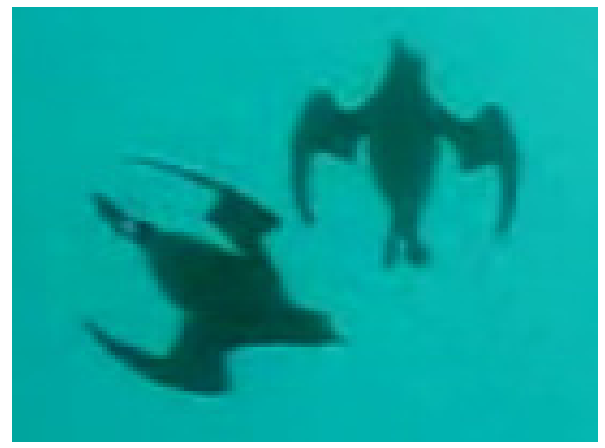

Figure 2: $\quad$ Guillemots wing shape whilst flapping under water [12].

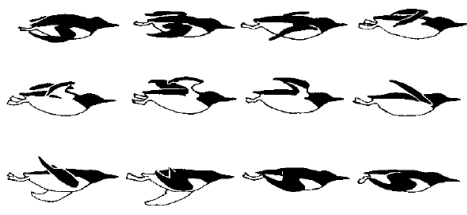

Figure 3: Wing position of common guillemot during horizontal forward swimming [9].

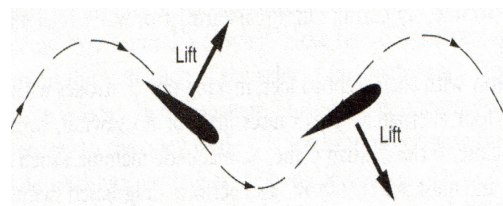

Figure 4: Wing path of a Humboldt penguin relative to the water, (thrust forces indicated by arrows) [2].

By analysing Figure 3, it would appear that the angle of attack of the guillemot indicates that substantial lift is created during the upstroke as well as the down stroke. This is illustrated in a more diagrammatic fashion in Figure 4.

The greater density of water obviously results in a change in the forces the guillemot must overcome for locomotion. Figure 5 shows the drag coefficient $C_{D-F r i}$ versus Reynolds number for a frozen common guillemot (body mass $=1.268 \mathrm{~kg}$, length $=0.444 \mathrm{~m}$, surface area $=0.0969 \mathrm{~m}^{2}$ ) and Figure 6 shows the values of drag from which the curve in $6 \mathrm{a}$ was derived.

In order to compare the power requirements for overcoming external forces in both air and underwater we must determine the forces experienced during underwater flapping. According to [15], the average speed of the common 
guillemot whilst flapping underwater is $1.52 \mathrm{~m} / \mathrm{s}$. Therefore if we extrapolate from Figure 6 we can calculate the approximate frictional drag experienced by the guillemot as: $D_{F r i}=0.5 \rho S_{w} U^{2} C_{D-F r i}$, where $\rho$ is the density of water $\left(\mathrm{kg} / \mathrm{m}^{3}\right)$, $S_{w}$ is the wetted surface area $\left(\mathrm{m}^{2}\right), U$ is the speed at which the bird is travelling $(\mathrm{m} / \mathrm{s})$ and $C_{D-F r i}$ is the frictional drag coefficient. From this, frictional drag at $1.52 \mathrm{~m} / \mathrm{s}$ is $1.864 \mathrm{~N}$. Note that drag from the wings is not reflected in this model.

It can be seen from the graph plotted in Figure 5 that at this speed of $1.52 \mathrm{~m} / \mathrm{s}$ the subsequent Reynolds number is approximately $1 \times 10^{6}$. Therefore as the Re

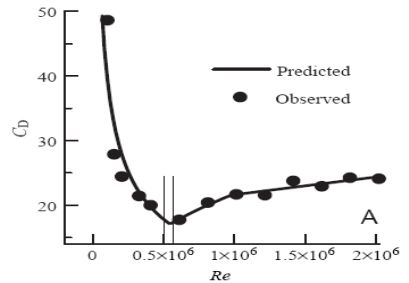

Figure 5:

Drag coef. $\left(\mathrm{C}_{\mathrm{D}}\right)$ vs.
Reynolds number
(Re) [15].

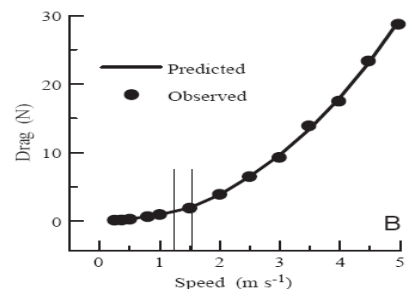

Figure 6: Observed values of drag producing the curve in 6a [15].

number increases, inertial forces dominate whilst the bird flaps through the water [15]. Additionally, the drag is dominated by pressure drag rather than skin friction drag which is the value calculated in Figure 6. In this situation, hydrodynamic efficiency can be enhanced by keeping the drag coefficients as small as possible, which is best achieved by streamlining the body shape. This therefore provides further reasoning behind the revised shape of the guillemots' wing; in order to improve the overall drag coefficients of their body shape they sweep their wings back to reduce their profile.

Existing literature has focused predominantly on frictional drag associated with body shape; less experimental data is available to model pressure drag associated with guillemots. Given that at this Re number pressure drag will represent the dominant force, we assume that the frictional drag will be $20 \%$ of the pressure drag, giving a pressure drag of $9.3 \mathrm{~N}$. By combining these values and multiplying by the average speed, the overall power requirements to overcome drag forces under water are:

$$
P_{\text {Sum }}=P_{F r i}+P_{\text {Pre }}=17.00 \mathrm{~W}
$$

Comparing this value to that obtained from the power required for flight in equation (7), and we can see that the values are very close in this situation. This indicates that the bird is operating at a similar level of exertion in both modes of locomotion, however not too much can be read into this very close correlation due to the assumptions that have been made when calculating the drag in water.

The guillemot is also capable of paddling on the water surface, using its feet as a means of propulsion. Although their ability based on the criteria set out in the previous section is only moderate compared with flapping in air and water, it does however play a crucial role in terms of providing a basis for takeoff and rest. This capacity will be analyzed in more detail in future work. 


\subsection{Guillemot: land}

Finally, it should be noted that the guillemot also has a limited level of ability on land, utilising this mode of locomotion as a means to rest and nest. Guillemots' typical breeding habitat is on cliff ledges and on offshore stacks [7]. Similarly to when the guillemot is on the water surface, the birds utilise the environmental features in order to help in getting airborne. Typically guillemots launch themselves from the cliffs so as to reach the minimum speed required for flight. However the birds' actual movement whilst on land is very limited.

\subsection{Overall effectiveness of the common guillemots}

We have now examined the various modes of locomotion of the common guillemot. Thus, by utilising the approach as detailed in Figure 1, we can rate the effectiveness of the different modes in one simple diagrammatic representation, detailed in Figure 7. Each type of locomotion within the various modes has been given a mark out of ten. Within this example there is certainly correlation between the types of locomotion that the guillemot is adapted for and the predominant methods used for locomotive purposes. This is a logical interaction but further research would be required to confirm this for all multi-modal animals. In addition to this measure of effectiveness there is the need for a measure of performance so that the individual abilities of different species can be cross-referenced. This is required so that when trying to select locomotion types and suitable combinations for engineering problems, design choices can be made based on animals using a standardised ranking system.

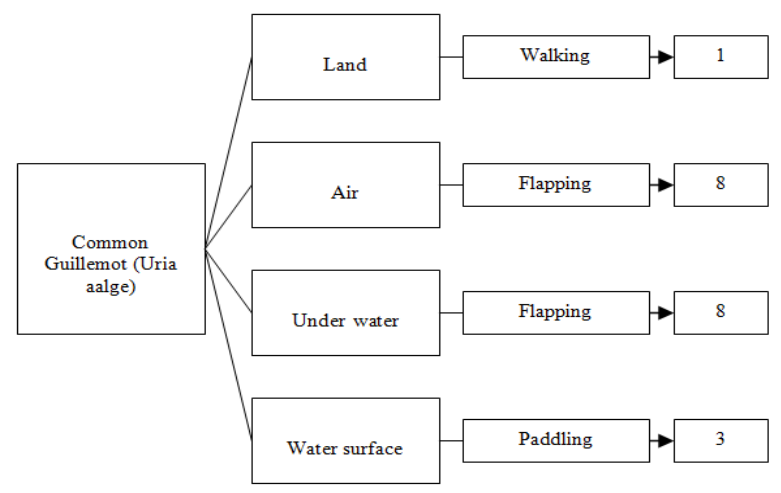

Figure 7: Diagrammatic representation of the effectiveness of the modes of locomotion for the common guillemot.

\subsection{Conclusion of analysis of the common guillemot}

On closer inspection of the various forms of modal locomotion it becomes very apparent that the types utilised by the guillemot are far from the best solution if 
they only travelled within a single mode. In reality the bird operates in various environments, with very different conditions between the substrates. What the guillemot has achieved is a good level of compromise between the different modes of locomotion, whilst still maintaining abilities in the substrates that help the animal survive. Much can still be learnt from further analysis of multi-modal animals in order to determine and understand other compromises that they exhibit.

\section{References}

[1] F. J. Boria, R. J. Bachmann, P. G. Ifju, R. D. Quinn, R. Vaidyanathan, C. Perry, J. Wagener, "A Sensor Platform Capable of Aerial and Terrestrial Locomotion," in IEEE Int. Conf. on Intel. Robots and Systems (IROS) Vancouver, CN, 2005.

[2] R. M. Alexander, Principles of animal locomotion. Princeton, N.J., USA: Princeton University Press, 2003.

[3] C. R. Taylor, et. al., "Energetics and mechanics of terrestrial locomotion, I: Metabolic energy consumption as a function of speed and body size in birds and mammals," J. of Exp. Bio., 97, 1-21, 1982.

[4] R. V. Baudinette, P. Gill, "The energetics of paddling and flying in water: Locomotion in penguins and ducks," J. of Comp. Physio, 155, 373-80, 1985

[5] http://news.bbc.co.uk/1/hi/uk/1405002.stm

[6] http://animals.nationalgeographic.com/animals/birds/ostrich.html

[7] A. J. Gaston, The Auks. Bird Families of the World (Series 5). Oxford, UK: Oxford University Press, 1998.

[8] C. J. Pennycuick, "Flight of Auks (Alcidae) and other northern seabirds compared with southern procellariiformes," J.of Exp. Bio., 128, 335-347, 1987.

[9] J. R. Lovvorn, "Upstroke thrust, drag effects, and stroke-glide cycles in wing-propelled swimming by birds," Amer. Zool, 41, 154-165, 2001.

[10] S. C. Burgess, Design and Nature: Course Notes, University of Bristol, 2008

[11] J. R. Lovvorn and et. al, "Hydrodynamic drag of diving birds: effects of body size, body shape and feathers at steady speeds" J. of Exp. Bio. 204, 1547-57, 2001.

[12] http://urgetosubmerge.vodpod.com/video/18979-diving-birds-at-rathlinisland

[13] D. E. Alexander, Nature's flyers: birds, insects and biomechanics of flight. Baltimore, MD, USA: Johns Hopkins University Press, 2002.

[14] C.A. Hui "Penguin swimming: Hydrodynamics" Phys Zool 61, 333-43, 1988

[15] J.R. Lovvorn et al. "Mechanical vs. physiological determinants of swimming speeds in diving Brunnicj's Guillemots," J. of Exp. Bio, 202, 1741-52, 1999 